\title{
Celdas de combustible microbiano acoplado a un reactor anaerobio de flujo ascendente de lecho fijo y fotocatálisis heterogéneo para el tratamiento de aguas mieles
}

\section{Microbial Fuel Cells Coupled with an Upflow Fixed Bed Anaerobic Reactor and a Heterogeneous Photocatalysis for the Treatment of Honey Water}

\author{
Bryan Santillan y Annghy Alvarez
}

Universidad Nacional Agraria de la Selva. Av. Universitaria s/n. Km 1.5, Tingo María.

Recibido el 28 de marzo del 2017. Revistado el 1 de julio del 2017. Aceptado el 20 de julio.

DOI: https://doi.org/10.33017/RevECIPeru2017.0005/

\section{Resumen}

En el presente trabajo se removió la materia orgánica de un efluente artificial de lavado de cacao usando Celdas de Combustible Microbiana Acopladas Al Reactor Anaeróbico De Flujo Ascendente de Lecho Fijo y junto con el Fotocatalizador Heterogéneo; también se redujo la carga microbiana, evitando la proliferación de microorganismos patógenos que intervienen en el incremento de la Demanda Bioquímica de Oxigeno, alteración biológica y reducción de la calidad en los cuerpos de agua, a la misma vez se midió la generación de energía en voltaje y amperaje producido en el RAFA. Para ello se caracterizaron las aguas residuales en la entrada, durante el proceso y en la salida; en la entrada y salida se midió la DBO5, sólidos totales disueltos, potencial de reducción, conductividad, $\mathrm{pH}$, temperatura y $\% \mathrm{NaCl}$, durante el proceso se midió el $\mathrm{pH}$, amperaje y voltaje. Seguidamente se obtuvo una tasa de remoción de DBO5 del $70.37 \%$ en promedio y según referencias bibliográficas la tasa de remoción de materia orgánica en sistemas de tratamiento secundario deben estar por encima del $70 \%$ de eficiencia. Por último se pudo concluir que el sistema integrado de tratamiento secundario y avanzado lograron una mayor eficiencia en la disminución de la materia orgánica y se pudo captar la generación $195.1 \mathrm{mV}$ y 116 uA como máximo durante los 6 días de monitorización en el RAFA con un volumen de 14.5 litros, obteniéndose una eficiencia de $1560.8 \mathrm{~mW} / \mathrm{m3}$.

Descriptores: Celdas de combustible microbiano, Reactor anaeróbico de flujo ascendentes de lecho fijo, Fotocatalizador heterogéneo, $\mathrm{DBO}_{5}, \mathrm{pH}$, Voltaje, Amperaje, tasa de remoción de materia orgánica.

\section{Abstract}

The current work removed the organic material from an artificial cacao washing effluent using microbial fuel cells coupled with an upflow fixed bed anaerobic reactor and a heterogeneous photocatalyst. Also, the the microbial charge was reduced, avoiding the proliferation of pathogenic micro organisms which interfere with the increase in the biochemical demand for oxygen, biological alteration and the reduction of quality in the bodies of water; at the same time, the generation of energy in volts and amps produced in the RAFA (acronym in Spanish) was measured. For this, the residual water at the entrance, during the process and at the exit was characterized. At the entrance and exit, the DBO5, total dissolved solids, reduction potential, conductivity, $\mathrm{pH}$, temperature and $\% \mathrm{NaCl}$ were measured; during the process the $\mathrm{pH}$, amperage and voltage were measured. A removal rate of DBO5 at an average of $70.37 \%$ was obtained and according to bibliographic references, the removal rate of organic material in secondary treatment systems should be above $70 \%$ efficiency. Finally, it can be concluded that the integrated secondary and advanced treatment system achieved a greater efficiency as the organic matter diminished and a maximum generation of $195.1 \mathrm{mV}$ and $116 \mathrm{uA}$ could be captured during the six days of monitoring in the RAFA; with a volume of 14.5 liters, obtaining an efficiency of $1560.8 \mathrm{~mW} / \mathrm{m} 3$.

Keywords: Microbial fuel cells, Upflow fixed bed anaerobic reactor, Heterogeneous photocatalyst, DBO (acronym in Spanish), pH, Voltage, Amperage, Removal rate of organic material. 


\section{Introducción}

La producción de café en el Perú ha sido muy variada a través de los años por las diferentes regiones caficultoras del país, sin embargo existe un problema ambiental que cada agricultor cafetalero es responsable de generarlo en el proceso del beneficio húmedo del café y cacao, ya que producen volúmenes incontables de aguas residuales conocidos como aguas mieles, las cuales se vierten directamente a los cuerpos de agua cercanas a sus plantas, mayormente sin alguna intervención de tratamiento previo. Este altera el equilibrio natural de los ambientes acuáticos, debido a la carga orgánica que lleva consigo, y diferentes características al agua natural por lo que consecuentemente llevaría a la extinción de las especies acuáticas que habitan en ella.

Por otro lado, el tratamiento de las aguas residuales presenta varios problemas como el consumo de considerables cantidades de energía eléctrica para los procesos de aireación y recirculación, el gran espacio ocupado por los reactores empleados, los costos derivados de la instalación de las dos tecnologías necesarias y los costos del almacenamiento de los lodos.

Desde hace varios años se sabe de la presencia de microorganismos en las aguas residuales que son capaces de degradar materia orgánica y generar corriente eléctrica. Las celdas de combustible microbianas (CCM), el nombre que se le ha dado al sistema que aprovecha las características de los mencionados microorganismos, podría proporcionar una respuesta a varios de los problemas mencionados anteriormente.

Al integrar el sistema fotocatalizador para el tratamiento previo de las aguas mieles se tendrá una gran reducción de materia orgánica e inorgánica y patógenos que permitirá la cuantificación de la actividad de los microorganismos eficaces nativos del suelo del BRUNAS con respecto al crecimiento microbiano y degradación de la carga orgánica en el RAFA, así la Temperatura se mantendrá en un mismo rango al realizar las pruebas y monitorización en el transcurso del día y en las noches se acoplara un sistema de calefacción, el pH se controlaría dentro del RAFA con $\mathrm{NaOH}$ para mantenerlo entre un rango de 5.5 a 6.5 para mantener las condiciones óptimas de degradación anaeróbica y realizar la medición de Voltaje por medio del sistema de Celdas de Combustible Microbiano.

El propósito de este sistema no sería competir con las tecnologías existentes para generación de electricidad a gran escala, sino tratar el agua residual y obtener durante el proceso un producto de valor agregado. Es decir, ver el tratamiento de aguas residuales como un proceso que valoriza la materia orgánica presente.

\section{Datos y metodología}

Para la realización de la presente investigación se requirió de un multiparámetro, multitester, peachimetro, oxímetro, balanza analítica y estufa para medir con rigurosidad los parámetros de caracterización de las aguas residuales de la industria del cacao y para la evaluación de cada tratamiento propuesto. También se usaron insumos como agar-agar, agar rugosa, carbón activado y antibióticos para el cultivo in vitro de microorganismos eficaces

\subsection{Reactor Anaeróbico de Flujo Ascendente con Lecho Fijo}

El diseño del Reactor Anaeróbico de Flujo Ascendente con lecho fijo tiene una altura de $1 \mathrm{~m}$ por que según investigaciones sobre RAFA o UASB (siglas en inglés) se demostró que a una altura menor, la producción de biogás es baja debido a la perdida de lodo, ya que el burbujeo aumenta al incrementarse la carga, y al tener un recorrido tan corto se impide la separación de las dos fases presentándose en algunos casos el lavado (salida) completo del reactor [3].

Para el muestreo a través del reactor, se diseñaron dos (2) puntos los cuales están distribuidos de la siguiente forma: de abajo hacia arriba, hay una apertura que está a $10 \mathrm{~cm}$ de la base, el cual permitirá la salida del cable de cobre que conecta al electrodo anódico, y de arriba hacia abajo a 20 $\mathrm{cm}$ se encuentra la llave de salida. En el punto de salida, se adoptó una tubería de PVC de 1/2" con una llave de apertura rápida del mismo diámetro. El equipo está rodeado de una espuma color negro, con el fin de lograr aislamiento térmico para mantener la temperatura a condiciones óptimas y para evitar la formación de algas y fitoplancton. Luego de que el agua pase por el RAFA, en la llave de salida se conectara a un balde de cinco (5) litros de capacidad pintado de color negro para evitar el ingreso de la luz directa, este balde tiene la función de almacenar en un tiempo prolongado el agua tratada del RAFA para poder establecer un nuevo caudal, finalmente se conectara al Fotocatalizor Heterogéneo mediante un tubo de PVC de 1/2",

\subsection{Celdas de Combustible Microbiano}


El diseño de la Celda de Combustible Microbiano utilizado para el acoplamiento al RAFA consta de 2 fases: anaeróbica y aeróbica, entre las cuales hay un separador que actuó como filtro y soporte fijo de piedra caliza combinado con teodolitos de PVC (1/2" de diámetro), que tendrá para la formación de biopelículas y transportador de electrones y protones, similar a una membrana de intercambio protónico (MIP). La fase anaeróbica está en el fondo del RAFA donde se colocó un electrodo que actuó como ánodo hecho de grafito cubierta con gaza en un soporte de madera esterilizada y la fase aeróbica se encuentra a la altura de la salida, que esta conecta al separador de gases-líquidos. El agua residual al oxidarse por acción de los microorganismos, generan electrones, protones, $\mathrm{CO} 2$ y CH4; para evitar la acumulación de gases, se construyó una campana separador de gas el cual está conectada a la zona de recogida de gases, que consta de un recipiente lleno de agua para evitar el lavado en los lechos y manto generado en el RAFA. En la parte superior después del soporte fijo se colocó un electrodo de grafito cubierto con gaza que actuara como cátodo el cual fue aireado en forma pasiva, una vez los electrones se liberan en el fondo del reactor (parte anódica), éstos son captados por el ánodo y posteriormente transferidos hacia el cátodo mediante un circuito externo (cableado de cobre). Simultáneamente, en la cámara anódica se generan protones que migran hacia la cámara catódica a través del separador que será el soporte fijo, donde se combinan con el oxígeno generado con la aireación de una bomba pequeña, para reducirse a agua con los electrones que captan directamente del cátodo.

\subsection{Fotocatalizador Heterogéneo}

El Fotocatalizador se construyó una estructura de madera con dimensiones de $40 \mathrm{~cm} \times 60 \mathrm{~cm} \times 5 \mathrm{~cm}$ donde se colocaron tubos de PVC cortados por la mitad del diámetro. El flujo del agua a tratar al pasar por los conductos tendrá contacto con el TiO2 que estará cubierto en la superficie del canal. Para el funcionamiento y operación fue mediante una llave de paso que se gradúo el caudal de salida de agua residual desde el tanque de alimentación que estará conectado al primer canal donde se le aplicara el TiO2 como catalizador, tienen que estar a dirección del sol.

\subsection{Preparación de las muestras}

Se realizó la preparación de 40 L. de agua simulada en la cual se lavó con $5.5 \mathrm{~kg}$ de cacao (55gr de melaza), se dejó 21 días de fermentación para simular el estancamiento en zonas afectadas, de esta agua se utilizará $1 \mathrm{~L}$. para realizar los análisis de los parámetros de entrada las cuales fueron el DBO5, OD, Solidos disueltos totales, Temperatura y $\mathrm{pH}$. Para las mediciones de OD, temperatura, conductividad y $\mathrm{pH}$ se utilizará un equipo multiparámetro y para determinar los sólidos totales y DBO5, se realizarán las mediciones en laboratorio.

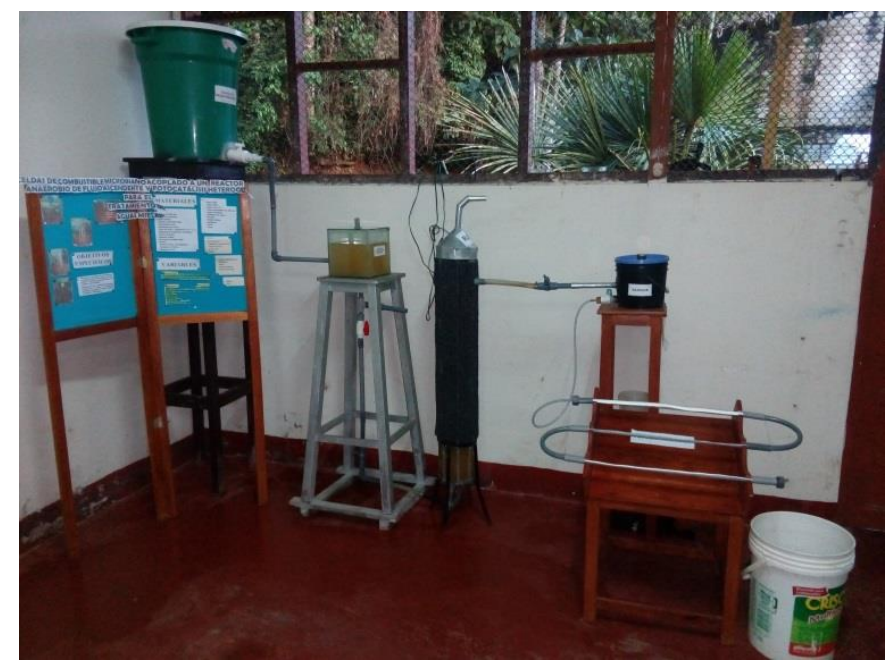

Figura 1. Sistema integrado del Reactor Anaeróbico de Flujo Ascendente con Lecho Fijo y Fotocatalizador Heterogéneo.

\section{Resultados y discusión}

3.1. Variación de los parámetros de $\mathrm{pH}$, voltaje y amperaje durante la monitorización del sistema.

Los parámetros monitorizados a lo largo del trabajo muestran que para el $\mathrm{pH}$ de la cámara de aclimatación se encontraba en un intervalo de 4.6 4.78 (Considerando que se realizó un control de este parámetro utilizando $\mathrm{NaOH}$ ), con respecto a la temperatura se mantuvo en el intervalo de 24.9 $26.6{ }^{\circ} \mathrm{C}$, estando dentro del rango de una temperatura ambiente donde los microorganismo tienen una actividad estable, estas mediciones se hizo con un termómetro (control y verificación) y con el uso del multiparámetro del Laboratorio de Calidad y Tratamiento de Suelos de la Universidad Nacional Agraria de la Selva. 


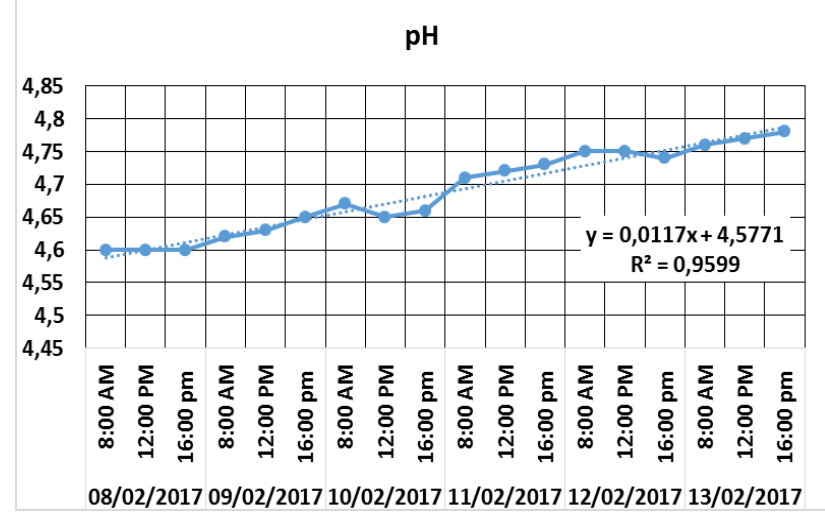

Figura 2. Variación del $\mathrm{pH}$ en la cámara de acondicionamiento.

Para la variación del $\mathrm{pH}$ en la cámara de acondicionamiento se puede observar (Figura 2) que en la etapa de aclimatación existen diversos picos en los que el $\mathrm{pH}$ disminuye y aumenta a lo largo de toda esta etapa (comportamiento regulado con aplicaciones de $\mathrm{NaOH}$ ).

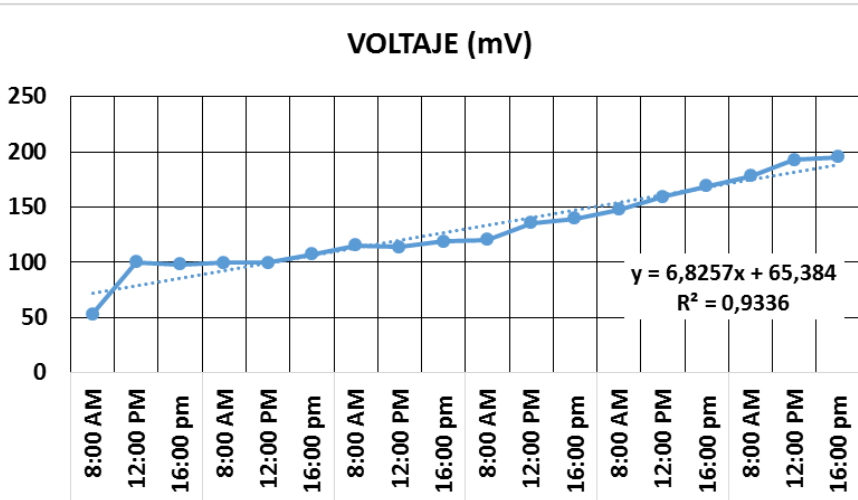

08/02/2017 09/02/2017 10/02/2017 11/02/2017 12/02/2017 13/02/2017

Figura 3. Variación del voltaje en el RAFA de lecho fijo.

Se observa (Figura 3) que durante las primeras pruebas el voltaje en el Reactor Anaeróbico de Flujo Ascendente de Lecho se obtuvo en la etapa de aclimatación, como se observa en la figura 1 una tendencia de aumentar el pH según incrementa la degradación de la materia orgánica.

Mientras mayor degradación de la materia orgánica a través de la actividad microbiana (consorcios entre geobarcter, levaduras facultativas, etc.), mayor flujo de electrones se registraran en el medio acuoso; para esto se debe mantener un registro continuo en periodos largos para encontrar el umbral del registro de voltaje y correlacionarlo con la biomasa presente para determinar cualitativa y cuantitativamente la biodegradabilidad de los contaminantes orgánicos presentes en el agua residual de cacao.

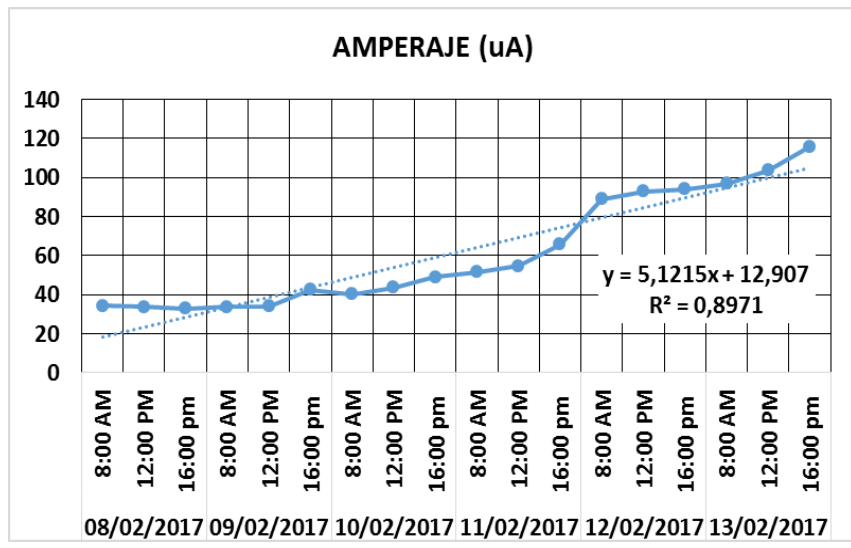

Figura 4. Amperaje (uA) en RAFA de Lecho Fijo

En la Figura 4 se observa que durante las primeras pruebas el amperaje en el Reactor Anaeróbico de Flujo Ascendente de Lecho se obtuvo en la etapa de aclimatación (como se observa en el Figura 3) una tendencia de aumentar según incremente la degradación de la materia orgánica, ya que tiene una correlación dependiente con el voltaje.

\subsection{Diseño del sistema de tratamiento de aguas mieles.}

Para realizar un tratamiento eficaz, se tuvo que graduar el TRH para realizar la mezcla continua de los microorganismos eficaces que se aclimataban en la cámara de acondicionamiento microbiano, esto permitió evaluar la evolución de la actividad microbiana y la retención de estos mismos antes de ser tratados con el Fotocatalizador heterogéneo.

Tabla 1. Diseño del sistema de tratamiento.

\begin{tabular}{lccc}
\hline \multicolumn{1}{c}{ EQUIPOS } & $\begin{array}{c}\text { VOLUMEN } \\
\text { (I) }\end{array}$ & $\begin{array}{c}\text { TRH } \\
\text { (horas) }\end{array}$ & $\begin{array}{c}\text { CAUDAL } \\
\text { (ml/min) }\end{array}$ \\
\hline \hline $\begin{array}{l}\text { Tanque de } \\
\text { almacenamiento }\end{array}$ & 40 & 12 & 55.56 \\
$\begin{array}{l}\text { Cámara de } \\
\text { aclimatación }\end{array}$ & 8 & 2.25 & 59.26 \\
$\begin{array}{l}\text { RAFA de lecho } \\
\text { fijo }\end{array}$ & 14.5 & 12 & 20.14 \\
$\begin{array}{l}\text { Tanque de } \\
\text { recepción al } \\
\text { fotocatalizador } \\
\text { heterogéneo }\end{array}$ & 4 & 4.5 & 14.81 \\
\hline
\end{tabular}




\subsection{Determinación de la tasa de remoción de la materia orgánica.}

El Tabla 2, muestra los resultados obtenidos del DBO del agua de entrada y de las aguas de salida de una corrida que se realizaron durante el período de prueba del 08 hasta el 13 de febrero.

Tabla 2. Determinación del $\mathrm{DBO}_{5}$.

\begin{tabular}{|c|c|c|c|c|}
\hline $\begin{array}{c}\begin{array}{c}\text { Volumen } \\
\text { muestra } \\
(3.50 \%)\end{array} \\
\mathrm{T}\left({ }^{\circ} \mathrm{C}\right)\end{array}$ & $\begin{array}{l}\text { Agua des } \\
\frac{\text { Agua de m }}{\text { Inicia }}\end{array}$ & iestra & $\begin{array}{r}19.2 \\
\text { Tratan }\end{array}$ & $\frac{1}{n+0}$ \\
\hline \multirow{3}{*}{26} & $\begin{array}{l}\mathrm{DBO}_{5} \\
(\mathrm{mg} / \mathrm{l})\end{array}$ & $\begin{array}{c}188.4 \\
1 \\
\end{array}$ & $\begin{array}{l}\mathrm{DBO}_{5} \\
(\mathrm{mg} / \mathrm{l})\end{array}$ & $\begin{array}{c}55.8 \\
2 \\
\end{array}$ \\
\hline & $\begin{array}{c}\mathrm{OD}_{\mathrm{ad}} \\
(\mathrm{mg} / \mathrm{l}) \\
\mathrm{OD}_{\mathrm{m}}(\mathrm{mg} / \mathrm{l})\end{array}$ & 6.83 & $\begin{array}{c}\mathrm{OD}_{\mathrm{ad}} \\
(\mathrm{mg} / \mathrm{l}) \\
\mathrm{OD}_{\mathrm{m}} \\
(\mathrm{mg} / \mathrm{l})\end{array}$ & 6.73 \\
\hline & $\begin{array}{l}\mathrm{OD}_{\mathrm{adf}} \\
(\mathrm{mg} / \mathrm{l}) \\
\mathrm{OD}_{\mathrm{mf}} \\
(\mathrm{mg} / \mathrm{l})\end{array}$ & 6.88 & $\begin{array}{l}\mathrm{OD}_{\mathrm{adf}} \\
(\mathrm{mg} / \mathrm{l}) \\
\mathrm{OD}_{\mathrm{mf}} \\
(\mathrm{mg} / \mathrm{l})\end{array}$ & 6.71 \\
\hline
\end{tabular}

El Tabla 3, muestra los resultados obtenidos por medio del multiparámetro electrónico del laboratorio de calidad y tratamiento de suelos de la Universidad Nacional Agraria de la Selva.

Tabla 3. Determinación de las variables representativas.

\begin{tabular}{|c|c|c|c|c|c|c|}
\hline $\begin{array}{c}\text { EQUIP } \\
0\end{array}$ & $\begin{array}{c}\mathrm{T} \\
\left({ }^{\circ} \mathrm{C}\right)\end{array}$ & $\mathrm{pH}$ & $\begin{array}{l}\mathrm{DBO}_{5} \\
(\mathrm{mg} / \mathrm{l})\end{array}$ & $\begin{array}{c}\text { STD } \\
\text { (ppm) }\end{array}$ & $\begin{array}{l}\text { Cond } \\
\text {. (uS) }\end{array}$ & $\begin{array}{l}\text { Pot. } \\
\text { Red. } \\
\text { (mV) } \\
\end{array}$ \\
\hline $\begin{array}{l}\text { Tanque } \\
\text { de } \\
\text { aliment } \\
\text { ación }\end{array}$ & 26.6 & 4.6 & 188.41 & $\begin{array}{c}489000 \\
0\end{array}$ & 9.76 & 132.7 \\
\hline $\begin{array}{l}\text { Cámar } \\
\text { a de } \\
\text { aclimat } \\
\text { ación }\end{array}$ & 24.9 & 4.78 & - & $\begin{array}{c}489000 \\
0\end{array}$ & 0.03 & $\begin{array}{r}132.7 \\
121.8\end{array}$ \\
\hline $\begin{array}{l}\text { RAFA } \\
\text { de } \\
\text { lecho } \\
\text { fijo }\end{array}$ & 24.7 & 4.98 & 55.82 & 0.02 & 0.04 & 111.3 \\
\hline $\begin{array}{l}\text { Fotocat } \\
\text { álisis } \\
\text { heterog } \\
\text { éneo }\end{array}$ & 24.9 & 5.1 & & 0.01 & 0.03 & 104.4 \\
\hline
\end{tabular}

\subsection{Eficiencia del Potencial Coulombimétrica}

La $n_{d b o}$ se cuantifico para la remoción de materia orgánica del sistema, se utiliza el DQO o DBO para determinar la eficiencia coulombimétrica que es realizada por la actividad de los microorganismos eficaces en degradar la materia orgánica y producción electrones por la oxidación que son transportados por solución electrolítica del medio:

Se tiene como valor un $70.37 \%$ de eficiencia coulombimétrica a través de la siguiente formula:

$$
n_{d b o}=\frac{D B O_{\text {inicial }}-D B O_{\text {final }}}{D B O_{\text {final }}} \times 100
$$

Con esta fórmula también se determina el porcentaje de reducción la materia orgánica presente.

\subsection{Diseño Experimental Box-Benkhen.}

Tabla 4. Datos del RAFA de lecho fijo.

\begin{tabular}{cccc}
\hline \multicolumn{4}{c}{ RAFA DE LECHO FIJO } \\
$\mathbf{T}{ }^{\circ} \mathbf{C}$ & $\mathbf{p H}$ & $\begin{array}{c}\text { VOLTAJE } \\
(\mathbf{m V})\end{array}$ & $\begin{array}{c}\text { OD } \\
(\mathbf{m g} / \mathbf{l})\end{array}$ \\
\hline 24.7 & 4.6 & 53.1 & 2.515 \\
26.6 & 4.6 & 115.3 & 2.515 \\
24.7 & 4.98 & 178.1 & 2.515 \\
26.6 & 4.98 & 195.1 & 2.515 \\
24.7 & 4.79 & 100.3 & 0.28 \\
26.6 & 4.79 & 168.9 & 0.28 \\
24.7 & 4.79 & 107.2 & 4.75 \\
26.6 & 4.79 & 118.8 & 4.75 \\
25.65 & 4.6 & 114 & 0.28 \\
25.65 & 4.98 & 192.8 & 0.28 \\
25.65 & 4.6 & 99.6 & 4.75 \\
25.65 & 4.98 & 159.6 & 4.75 \\
25.65 & 4.79 & 147.5 & 2.515 \\
25.65 & 4.79 & 139.8 & 2.515 \\
25.65 & 4.79 & 135.61 & 2.515 \\
\hline
\end{tabular}

La Tabla 4, muestra los datos registrados durante los días de monitorización del Reactor Anaeróbico de Flujo Ascendente con lecho fijo, donde para realizar el diseño de Box-Benkhen se deben seleccionar los datos mínimos, máximos e intermedios de las variables de entrada.

Las variables de entrada fueron la temperatura, el $\mathrm{pH}$ y el oxígeno disuelto (OD) y la variable de salida fue el Voltaje $(\mathrm{mV})$, el cual se usaran los valores mínimos, máximos e intermedios.

\subsection{Análisis de Varianza para Voltaje}


Tabla 5. Análisis de varianza para voltaje

\begin{tabular}{|c|c|c|c|c|c|}
\hline Fuente & $\begin{array}{l}\text { Suma de } \\
\text { cuadrad } \\
\text { os }\end{array}$ & & $\begin{array}{l}\text { Cuadrad } \\
\text { o Medio }\end{array}$ & $\begin{array}{l}\text { Razón- } \\
\text { F }\end{array}$ & Val \\
\hline $\begin{array}{l}\text { A:Tempe } \\
\text { ratura }\end{array}$ & 3176.04 & $\overline{11}$ & 3176.04 & 25.62 & 0.0039 \\
\hline B:pH & 14757.6 & 1 & 14757.6 & 119.05 & 0.0001 \\
\hline C:OD & 1030.58 & 1 & 1030.58 & 8.31 & 0.0345 \\
\hline AA & 499.84 & 1 & 499.84 & 4.03 & 0.1009 \\
\hline AB & 510.76 & 1 & 510.76 & 4.12 & 0.0981 \\
\hline$A C$ & 812.25 & 1 & 812.25 & 6.55 & 0.0507 \\
\hline BB & 135.819 & 1 & 135.819 & 1.10 & 0.3432 \\
\hline BC & 88.36 & 1 & 88.36 & 0.71 & 0.4370 \\
\hline CC & 113.118 & 1 & 113.118 & 0.91 & 0.3833 \\
\hline $\begin{array}{l}\text { Error } \\
\text { total }\end{array}$ & 619.784 & 5 & 123.957 & & \\
\hline $\begin{array}{l}\text { Total } \\
\text { (corr.) }\end{array}$ & 21773.7 & 14 & & & \\
\hline
\end{tabular}

Se tiene un R-cuadrada igual a $97.1535 \%$, Rcuadrada (ajustada por g.l.) igual a $92.0298 \%$, el error estándar del est. es 11.1336, el error absoluto medio es 5.41733, el estadístico Durbin-Watson es igual a $2.45005(\mathrm{P}=0.6127)$ y la autocorrelación residual de Lag 1 es -0.301461 .

La tabla ANOVA particiona la variabilidad de Voltaje en piezas separadas para cada uno de los efectos. Entonces prueba la significancia estadística de cada efecto comparando su cuadrado medio contra un estimado del error experimental.

En este caso, 3 efectos tienen una valor-P menor que 0.05 , indicando que son significativamente diferentes de cero con un nivel de confianza del $95.0 \%$. \%.

El error estándar del estimado muestra que la desviación estándar de los residuos es 11.1336. El error medio absoluto (MAE) de 5.41733 es el valor promedio de los residuos.

El estadístico de Durbin-Watson (DW) prueba los residuos para determinar si haya alguna correlación significativa basada en el orden en que se presentan los datos en el archivo.

Puesto que el valor-P es mayor que $5.0 \%$, no hay indicación de autocorrelación serial en los residuos con un nivel de significancia del 5.0\%

Estos datos nos servirán para graficar el diagrama de Pareto Estandarizada y ver así que variables tienen una mayor relación para determinar los valores óptimos del voltaje según la gráfica de superficie de respuesta que nos brindara el programa de Statgraphics.

\subsection{Camino de Máximo Ascenso para Voltaje}

Tabla 6. Camino de máximo ascenso para voltaje

\begin{tabular}{cccc}
\hline $\begin{array}{c}\text { Temperatura } \\
\left({ }^{\circ} \mathbf{C}\right)\end{array}$ & $\begin{array}{c}\mathbf{p H} \\
([\mathbf{H}+])\end{array}$ & $\begin{array}{c}\text { OD } \\
(\mathbf{m g} / \mathbf{l})\end{array}$ & $\begin{array}{c}\text { Voltaje } \\
(\mathbf{m V})\end{array}$ \\
\hline \hline 25.65 & 4.79 & 2.515 & 140.97 \\
26.2147 & 4.75594 & 3.515 & 132.803 \\
26.182 & 4.66042 & 4.515 & 107.463 \\
26.1671 & 4.59511 & 5.515 & 87.8557 \\
26.1993 & 4.5528 & 6.515 & 70.3036 \\
26.2962 & 4.52805 & 7.515 & 51.7689 \\
\hline \hline
\end{tabular}

Este es el trayecto, desde el centro de la región experimental actual, a través del cual la respuesta estimada cambia más rápidamente con un cambio menor en los factores experimentales.

Indica buenas características para ejecutar experimentos adicionales si el objetivo es incrementar o decrementar el voltaje. Actualmente, 6 puntos se han generado cambiando OD en incrementos de $1.0 \mathrm{mg} / \mathrm{l}$.

\subsubsection{Optimizar Respuesta}

Se pretende maximizar el Voltaje obteniéndose como óptimo $211.741 \mathrm{mV}$

Tabla 7. Valores optimizados

\begin{tabular}{llll}
\hline Factor & Bajo & Alto & Óptimo \\
\hline \hline Temperatura & 24.7 & 26.6 & 26.5838 \\
pH & 4.6 & 4.98 & 4.98 \\
OD & 0.28 & 4.75 & 0.280305 \\
\hline
\end{tabular}

Esta tabla muestra la combinación de los niveles de los factores, la cual maximiza Voltaje sobre la región indicada.Puede establecer el valor de uno o más factores a una constante, estableciendo los límites alto y bajo en ese valor. Una vez obtenido los valores óptimos a través del diseño BoxBenkhen usando el programa Statgraphics, se debe realizar la validación por medio de la experimentación contralando las variables de entrada por un periodo largo y así usar modelos estadísticos que prueben la correlación y verifiquen los datos propuestos por el diseño; también se podrían proponer nuevos tratamientos para alcanzar mayores valores. 


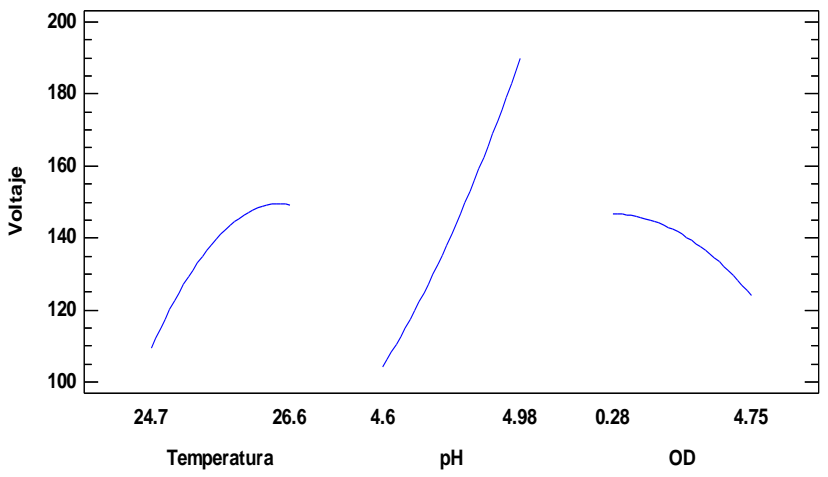

Figura 5. Efectos principales de voltaje

En la figura 5 se puede observación la relación de las variables de entrada ( $\mathrm{pH}$, Temperatura y $\mathrm{OD})$ con respectos a la variable de salida (Voltaje) donde presentan correlaciones no lineales y los puntos máximos y bajos según los datos introducidos al programa.

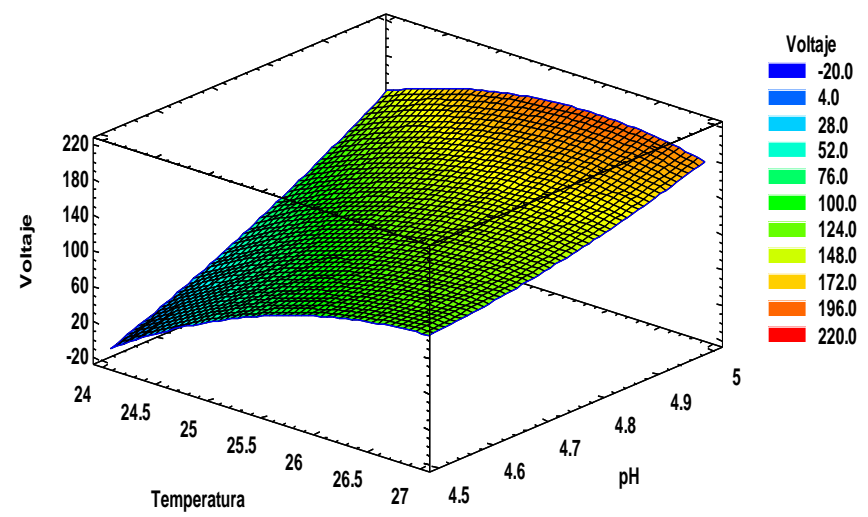

Figura 6. Superficie de Respuesta estimada Voltaje $-T^{o}-p H$.
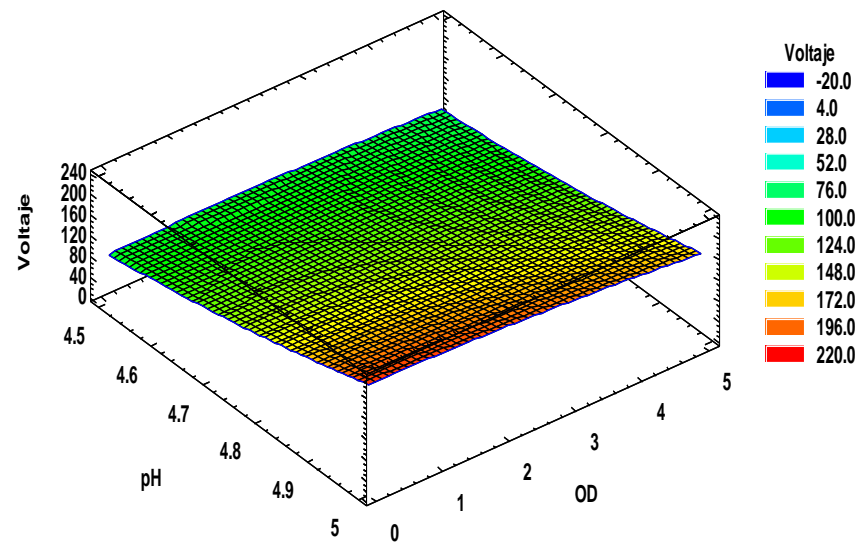

Figura 7. Superficie de Respuesta estimada Voltaje $-p H-O D$.

En la figura 6 y 7 se muestra el grafico de superficie de respuesta del Voltaje $(\mathrm{mV})$

\subsection{Diseño Experimental Box-Benkhen}

Tabla 8. Datos de lecho fijo para Amperaje.

\begin{tabular}{cccc}
\hline \multicolumn{4}{c}{ RAFA DE LECHO FIJO } \\
$\mathbf{T}$ & $\mathbf{p H}$ & $\begin{array}{c}\text { AMPERAJE } \\
\text { (uA) }\end{array}$ & OD (mg/l) \\
\hline 24.7 & 4.6 & 34 & 2.515 \\
26.6 & 4.6 & 42.4 & 2.515 \\
24.7 & 4.98 & 97 & 2.515 \\
26.6 & 4.98 & 116 & 2.515 \\
24.7 & 4.79 & 43.5 & 0.28 \\
26.6 & 4.79 & 89 & 0.28 \\
24.7 & 4.79 & 48.9 & 4.75 \\
26.6 & 4.79 & 93 & 4.75 \\
25.65 & 4.6 & 40.2 & 0.28 \\
25.65 & 4.98 & 103.8 & 0.28 \\
25.65 & 4.6 & 34.2 & 4.75 \\
25.65 & 4.98 & 94 & 4.75 \\
25.65 & 4.79 & 51.6 & 2.515 \\
25.65 & 4.79 & 54.6 & 2.515 \\
25.65 & 4.79 & 65.8 & 2.515 \\
\hline
\end{tabular}

Las variables de entrada fueron la temperatura, el $\mathrm{pH}$ y el oxígeno disuelto (OD)

\subsection{Análisis de Varianza para AMPERAJE}

La tabla ANOVA particiona la variabilidad de AMPERAJE en piezas separadas para cada uno de los efectos. Entonces prueba la significancia estadística de cada efecto comparando su cuadrado medio contra un estimado del error experimental. En este caso, 2 efectos tienen una valor-P menor que 0.05 , indicando que son significativamente diferentes de cero con un nivel de confianza del $95.0 \%$.

Tabla 9. Datos de lecho fijo para Amperaje

\begin{tabular}{|c|c|c|c|c|c|}
\hline Fuente & $\begin{array}{c}\text { Suma de } \\
\text { Cuadrad } \\
\text { os }\end{array}$ & GI & $\begin{array}{l}\text { Cuadrad } \\
\text { o Medio }\end{array}$ & $\begin{array}{c}\text { Razón } \\
\text {-F }\end{array}$ & \\
\hline A:T & 1711.13 & 1 & 1711.13 & 12.28 & 0.0172 \\
\hline B:p & 8450.0 & 1 & 8450.0 & 60.64 & 0.0006 \\
\hline C:OD & 5.12 & 1 & 5.12 & 0.04 & 0.8555 \\
\hline AA & 223.681 & 1 & 223.681 & 1.61 & 0.2610 \\
\hline$A B$ & 28.09 & 1 & 28.09 & 0.20 & 0.6722 \\
\hline AC & 0.49 & 1 & 0.49 & 0.00 & 0.9550 \\
\hline BB & 193.186 & 1 & 193.186 & 1.39 & 0.2920 \\
\hline BC & 3.61 & 1 & 3.61 & 0.03 & 0.8784 \\
\hline CC & 44.801 & 1 & 44.801 & 0.32 & 0.5952 \\
\hline \multirow{2}{*}{$\begin{array}{l}\text { Error total } \\
\text { Total } \\
\text { (corr.) }\end{array}$} & 696.792 & 5 & 139.358 & & \\
\hline & 11304.1 & 14 & & & \\
\hline
\end{tabular}


El estadístico R-Cuadrada indica que el modelo, así ajustado, explica $93.8359 \%$ de la variabilidad en AMPERAJE y el ajustada, que es más adecuado para comparar modelos con diferente número de variables independientes, es $82.7406 \%$. El error estándar del estimado muestra que la desviación estándar de los residuos es 11.805 . El error medio absoluto (MAE) de 6.11556 es el valor promedio de los residuos. El estadístico de Durbin-Watson es 2.3461 que prueba los residuos para determinar si haya alguna correlación significativa basada en el orden en que se presentan los datos., el valor-P es 0.5362 y es mayor que $5.0 \%$, no hay indicación de autocorrelación serial en los residuos con un nivel de significancia del $5.0 \%$.

Estos datos nos servirán para graficar el diagrama de Pareto estandarizada y ver así que variables tienen una mayor relación para determinar los valores óptimos del amperaje según las grafica de superficie de respuesta.

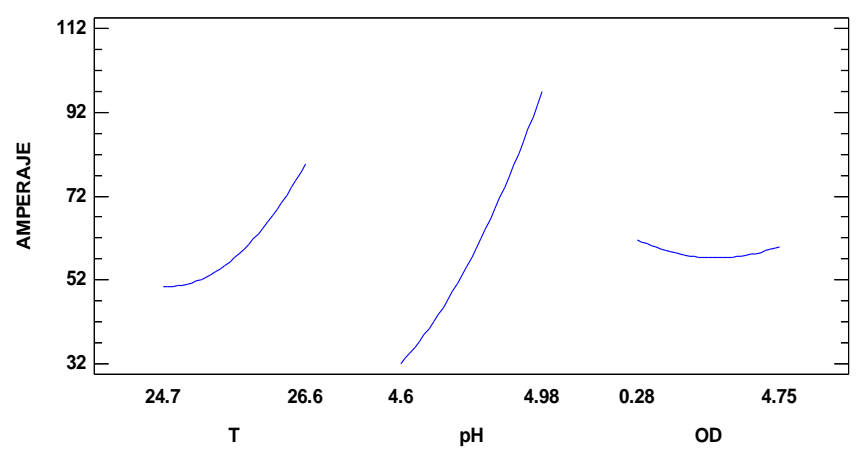

Figura 8. Efectos principales de amperaje

En la figura 8 se puede observación la relación de las variables de entrada $(\mathrm{pH}$, Temperatura y OD) con respectos a la variable de salida (Amperaje) donde presentan correlaciones no lineales.

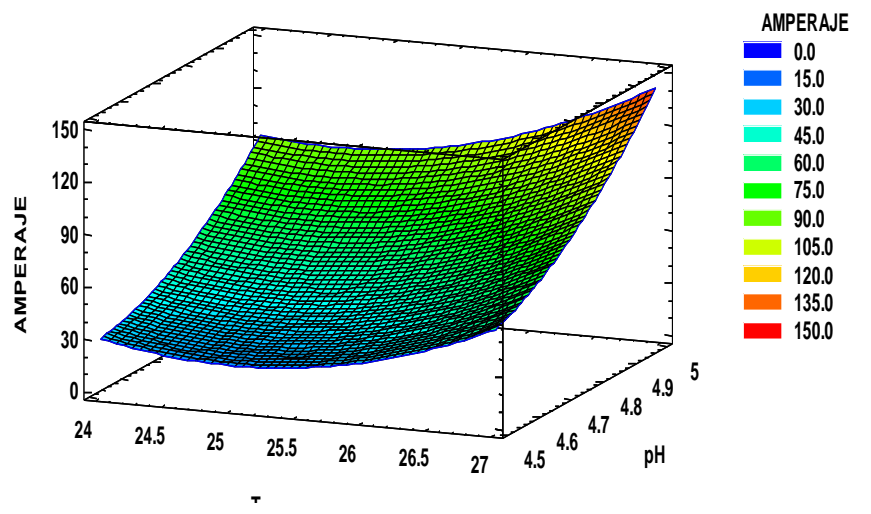

Figura 9. Superficie de Respuesta estimada Amperaje - $T^{o}-p H$.

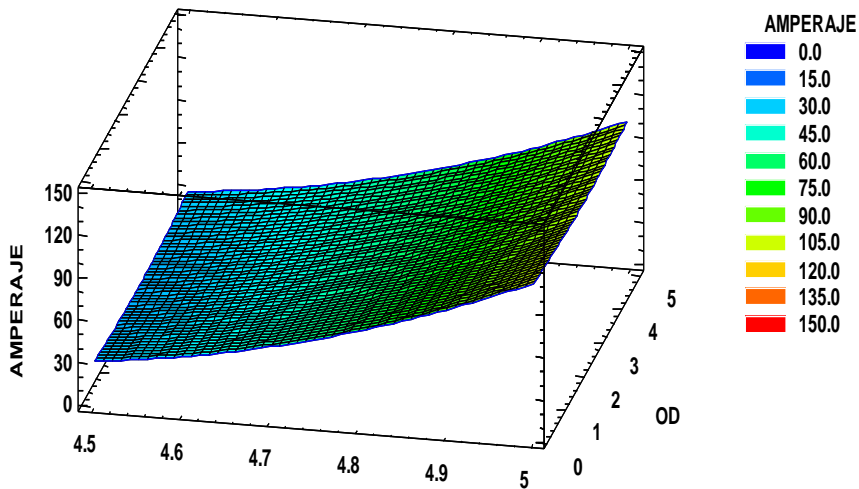

$\mathrm{pH}$

Figura 10. Superficie de Respuesta estimada Amperaje-pH-OD.

En la figura 9 y 10 se muestra el grafico de superficie de respuesta el cual es el fin del diseño Box-Benkhen, en él se muestran gráficos en 3D.

En el análisis de los resultados de voltaje y amperaje se tiene como máximo $195.1 \mathrm{mV}$ y 116 $\mathrm{uA}$ en un volumen de 14.5 litros (capacidad total del RAFA) y una potencia de $1560.8 \mathrm{~mW} / \mathrm{m3}$.

En los resultados obtenidos sobre los parámetros de $\mathrm{pH}$, amperaje y voltaje durante la etapa de aclimatación, se puede apreciar que el pH estuvo en aumento a lo largo de la monitorización (regulándose con $\mathrm{NaOH}$ en el caso de que el pH estuviera fuera del rango de tratamiento). La fermentación ácida durante la digestión anaeróbica tiende a bajar el $\mathrm{pH}$, debido a la producción de ácidos grasos volátiles (AGVs) y otros productos intermediarios [7]; también se pudo apreciar que la cámara de acondicionamiento microbiano presentó un comportamiento oscilatorio mínimo con tendencia incremental durante la etapa de aclimatación (Figura 1), esto se debe a que el primer paso para biodegradar sustancias en una planta de tratamiento de aguas residuales se debe a la aclimatación de los microorganismos [1]. Cuando los microorganismos se ponen en contacto con compuestos tóxicos, en un ambiente favorable, la aclimatación a estos compuestos puede ocurrir. Diversos fenómenos se han propuesto para explicar la fase de la aclimatación. Existe una selección y multiplicación de microorganismos especializados durante esta fase, además pueden existir transformaciones fisiológicas en el sistema metabólico de los microorganismos, es decir, alteraciones a nivel de regulación y producción enzimática, mutaciones, etc., es por estas razones que durante esta aclimatación la generación de ácidos grasos volátiles (AGVs) y otros productos intermediarios deben ser menor durante las pruebas realizadas [5]; en las pruebas realizadas no se mostraron una disminución del $\mathrm{pH}$ en el 
transcurso de los días monitorizados, al contrario se generó un incremento paulatino.

El comportamiento del $\mathrm{pH}$ en la parte aeróbica y anaeróbica de un biorreactor, en este caso del Reactor Anaeróbico de Flujo Ascendente de lecho fijo se debe a la diferencia de la velocidad de reacción de protones, electrones y oxígeno, siendo así; si no existe una diferencia significativa entre ambas velocidades el flujo de protones seria discontinuo y no existiría una acumulación de estos en la parte aeróbica, pero si la velocidad de generación de protones en la parte anaeróbica supera la velocidad de reacción en la parte aeróbica entonces los protones se acumularan en dicha zona por lo que se generará un pH más ácido [6]; esto se puede confirmar con el análisis en la zona anaeróbica y no en la cámara de acondicionamiento microbiano como se muestra en los resultados obtenidos que registran el aumento de pH según la aclimatación y por la recirculación del agua tratada. Los valores del voltaje y amperaje solo variaron durante las pruebas realizadas, a diferencia de la etapa de aclimatación (como se observa en el Figura 2) donde los resultados obtenidos del voltaje en el Reactor Anaeróbico de Flujo Ascendente de lecho fijo fueron incrementando paulatinamente, ya que durante el proceso de tratamiento existió buena actividad microbiana, y considerando que el diseño de las celdas de combustible microbiano captan los electrones producidos por la biodegradación del sustrato que directamente lo registra como electricidad, en el trabajo se apreció el incremento en valores de voltaje y amperaje.

Los resultados de DBO muestran una relación inversa en la generación de voltaje y amperaje, donde la menor DBO presenta mayor porcentaje de remoción (mayor a 1.5 de OD), lo que concuerda con "La cantidad de materia orgánica removida (So-S) (...) en el sistema es proporcional a la cantidad de materia orgánica remanente", es decir, el porcentaje de remoción de materia orgánica es inversamente proporcional a la cantidad de materia orgánica empleada (no remanente) expresada mediante la DBO obtenida después de cada corrida [4]. Además, respecto al porcentaje de remoción el cual en promedio fue de $70.37 \%$; del cual, en una CCM se logra medir su porcentaje de remoción, resultando en un $70 \%$ del carbono orgánico; si bien es cierto esta remoción es medida mediante COT y trabaja con una sola cámara, también prescinde el uso de membrana de intercambio protónico; ambos resultados de la remoción de materia orgánica (con carbono) presentan más de un $50 \%$ siendo buenos resultados [2]. Por último se puede estimar la eficiencia con la generación de watts por metros cúbicos según sea la capacidad de volumen tratado en el sistema, muchas referencias sobre el CCM, buscan nuevos materiales, insumos y procesos que mejoren la eficiencia en $\mathrm{W} / \mathrm{m} 3$.

Se Determinó que el aumento del pH en el sistema se debe al tratamiento completo de las aguas mieles; por otro lado, la variación del $\mathrm{pH}$ en la cámara de acondicionamiento como también en el RAFA se debe la diferencia entre la velocidad de reacción de protones, electrones y oxígeno y la velocidad de producción de protones en el ánodo. Las variaciones en la temperatura fueron generadas por las condiciones ambientales que se presentaron durante el monitoreo. $Y$ las variaciones en el Voltaje y Amperaje fueron producto de la actividad microbiana en la cámara anaeróbica del RAFA.

Para el aumento de la tasa de remoción de DBO mediante el acoplamiento de las celdas de combustible microbiano en el Reactor Anaeróbico de Flujo Ascendente de lecho fijo del cual se obtuvo un $70.37 \%$ en promedio, obteniendo una tasa de remoción de materia orgánica significativa, pues en promedio para resultar ser efectiva la remoción con un tratamiento secundario debe ser mayor al $70 \%$. Se pudo obtener una eficiencia de $1560.8 \mathrm{~mW} / \mathrm{m} 3$ siendo uno de los primeros trabajos realizados a laboratorio obteniéndose estos resueltos por el acoplamiento a un sistema de tratamiento secundario y avanzado, Reactor Anaeróbico de Flujo Ascendente de Lecho Fijo y Fotocatalizador Heterogéneo respectivamente.

\section{Conclusiones}

Se Determinó que el aumento del pH en el sistema se debe al tratamiento completo de las aguas mieles; por otro lado, la variación del $\mathrm{pH}$ en la cámara de acondicionamiento como también en el RAFA se debe la diferencia entre la velocidad de reacción de protones, electrones y oxígeno y la velocidad de producción de protones en el ánodo. Las variaciones en la temperatura fueron generadas por las condiciones ambientales que se presentaron durante el monitoreo. Y las variaciones en el Voltaje y Amperaje fueron producto de la actividad microbiana en la cámara anaeróbica del RAFA.

Para el aumento de la tasa de remoción de DBO mediante el acoplamiento de las celdas de combustible microbiano en el Reactor Anaeróbico de Flujo Ascendente de lecho fijo del cual se obtuvo un $70.37 \%$ en promedio, obteniendo una tasa de remoción de materia orgánica significativa, pues en 
promedio para resultar ser efectiva la remoción con un tratamiento secundario debe ser mayor al $70 \%$. Se pudo obtener una eficiencia de $1560.8 \mathrm{~mW} / \mathrm{m} 3$ siendo uno de los primeros trabajos realizados a laboratorio obteniéndose estos resueltos por el acoplamiento a un sistema de tratamiento secundario y avanzado, Reactor Anaeróbico de Flujo Ascendente de Lecho Fijo y Fotocatalizador Heterogéneo respectivamente.

\section{Agradecimiento}

A los catedráticos de diversos cursos como Física II, Ecología Aplicada, Biotecnología, Tratamiento de Contaminación de Suelos y Tratamiento de Aguas Residuales de la Universidad Nacional Agraria de la Selva (UNAS) por sus valiosos conocimientos que me permitió plantear esta investigación y apoyarme en el espacio (laboratorios), materiales, insumos, etc. y asesorarme sobre este tema innovador en mi universidad.

A mis compañeros Alex Segura, Elvis Medina y en especial a Annghy Alvarez que me apoyaron en la construcción y formulación de esta gran idea.

\section{Referencias}

[1] M. Aelion, C. Dobbins and K. Pfaender, Environmental Toxicology and Chemistry (1989), Vol. 8, p. 75.

[2] G. Buitron and J. Pérez, TIP Revista Especializada en Ciencias QuímicoBiológicas (2011) 5-11.

[3] F. Caicedo, Title thesis, Universidad Nacional de Colombia Sede Manizales, 2006.

[4] G. Valencia, Conceptos generales de tratamiento biológico (private communication).

[5] A. Wiggings, $H$. jones and A. Alexander, Applied and Environmental Microbiology (1987) 791-796.

[6] S. Yang, B. Jia and H. Liu, Bioresource Technology (2009), Vol 100, p. 1197-1202.

[7] A. Zehnder, American Society of Limnology and Oceanography (1988) 1-8. 